\title{
Almost-Interpolatory Chebyshev Quadrature
}

\author{
By K. Salkauskas*
}

Abstract. The requirement that a Chebyshev quadrature formula have distinct real nodes is not always compatible with the requirement that the degree of precision of an $n$ point formula be at least equal to $n$. This condition may be expressed as $\|d\|_{p}=0,1 \leqq p$, where $d=\left(d_{1}, \cdots, d_{n}\right)$ with

$$
d_{j}=\frac{\mu_{0}(\omega)}{n} \sum_{i=1}^{n} x_{i}^{i}-\mu_{j}(\omega), \quad j=1,2, \cdots, n,
$$

$\mu_{j}(\omega), j=0,1, \cdots$, are the moments of the weight function $\omega$ used in the quadrature, and $x_{1}, \cdots, x_{n}$ are the nodes. In those cases when $\|d\|_{2}$ does not vanish for a real choice of nodes, it has been proposed that a real minimizer of $\|d\|_{2}$ be used to supply the nodes. It is shown in this paper that, in such cases, minimizers of $\|d\|_{p}, 1 \leqq p<\infty$, always lead to formulae that are degenerate in the sense that the nodes are not all distinct. The results are valid for a large class of weight functions.

1. Introduction. In a recent paper [1], a new type of Chebyshev quadrature has been proposed. The results of numerical computations reported in that paper and intended to produce the nodes of these formulae show the apparent necessity of coincident nodes-in this sense, the formulae are degenerate. It will be shown that this degeneracy cannot be avoided.

The problem in [1] arises from the fact, proved by Bernstein [2], that it is impossible to find a real $n$-tuple $\left(x_{1}, \cdots, x_{n}\right)$ such that

$$
\int_{-1}^{1} f(x) d x=\frac{2}{n} \sum_{i=1}^{n} f\left(x_{i}\right)
$$

for all $f \in \mathcal{Q}_{n}$ when $n=8$ or $n>9$. If (1.1) is to hold for $\mathcal{P}_{n}$, it must hold for the basis $1, x, \cdots, x^{n}$ and conversely. This gives the following conditions on $x_{1}, \cdots, x_{n}$ :

$$
\frac{2}{n} \sum_{i=1}^{n} x_{i}^{i}=m_{i}, \quad j=0,1, \cdots, n,
$$

where

$$
m_{j}=\int_{-1}^{1} x^{j} d x= \begin{cases}2 /(j+1), & j \text { even }, \\ 0, & j \text { odd } .\end{cases}
$$

The first of Eqs. (1.2) is an identity, but the remaining equations cannot be satisfied by a real $n$-tuple if $n=8$ or $n>9$. The proposal in [1] is, then, to seek real $n$-tuples

Received August 17, 1970, revised October 5, 1971.

AMS (MOS) subject classifications (1970). Primary 41A55.

Key words and phrases. Chebyshev quadrature, minimum norm rules.

* This research was supported in part by the National Research Council of Canada, under Grant No. A-4050. 
$\zeta=\left(\zeta_{1}, \zeta_{2}, \cdots, \zeta_{n}\right)$, with $-1 \leqq \zeta_{1} \leqq \zeta_{2} \leqq \cdots \leqq \zeta_{n} \leqq 1$, that minimize the Euclidean norm of the residual vector $d=\left(d_{1}, \cdots, d_{n}\right)$, where

$$
d_{i}=\frac{2}{n} \sum_{i=1}^{n} x_{i}^{i}-m_{i}, \quad j=1,2, \cdots, n .
$$

For any $n$, if there exists a minimizer $\zeta$, the corresponding quadrature formula

$$
\int_{-1}^{1} f(x) d x=\frac{2}{n} \sum_{i=1}^{n} f\left(\zeta_{i}\right)+\text { error }
$$

will be said to be almost-interpolatory. The formula will be called degenerate if any two components of $\zeta$ are equal. For an interpolatory formula, the minimum value of $\|d\|_{2}$ is zero. Such formulae exist for $n<8$ and $n=9$ and are not degenerate. If the minimum value of $\|d\|_{2} \neq 0$, the formula is not interpolatory. Bernstein's result states that, for $n=8$ and $n>9,\|d\|_{2}$ cannot vanish; we shall prove that, in addition, a minimum of $\|d\|_{2}$ exists and yields only degenerate formulae.

2. Proof of Degeneracy. Let the mapping $G: R^{n} \rightarrow R^{n}$ be defined by

$$
G x=\left\{\begin{array}{c}
\frac{2}{n} \sum_{i=1}^{n} x_{i}-m_{1} \\
\vdots \\
\frac{2}{n} \sum_{i=1}^{n} x_{i}^{i}-m_{i} \\
\vdots \\
\frac{2}{n} \sum_{i=1}^{n} x_{i}^{n}-m_{n} .
\end{array}\right.
$$

By Bernstein's result, $G x=0$ has no roots in $R^{n}$ when $n=8$ or $n>9$. Let

$$
X=\left\{x \in R^{n}:-1 \leqq x_{1} \leqq x_{2} \leqq \cdots \leqq x_{n} \leqq 1\right\},
$$

and let $F$ be the restriction of $G$ to $X$. Then, $F x=0$ has no roots in $X$ when $n=8$ or $n>9$, and the proposal in [1] is to find $\zeta \in X$ such that $\|F \zeta\|_{2} \leqq\|F x\|_{2}$ for all $x \in X$.

It will be convenient to view $F$ as a composite mapping $F_{3} \cdot F_{2} \cdot F_{1}$, in which $F_{1}$ maps $x \in X$ onto the point $a=\left(a_{1}, \cdots, a_{n}\right)$ whose components are the coefficients of the polynomial $\tau^{n}+a_{1} \tau^{n-1}+\cdots+a_{n}$, having $x_{1}, \cdots, x_{n}$ as zeros. $F_{2}$ maps $a$ onto $s=\left(s_{1}, \cdots, s_{n}\right)$, the vector of sums of powers of the zeros, and is given by Newton's identities

$$
\begin{aligned}
& s_{1}+a_{1}=0 \\
& s_{2}+a_{1} s_{1}+2 a_{2}=0, \\
& \vdots \\
& s_{n-1}+a_{1} s_{n-2}+\cdots+(n-1) a_{n-1}=0, \\
& s_{n}+a_{1} s_{n-1}+\cdots+a_{n-1} s_{1}+n a_{n}=0 .
\end{aligned}
$$


Finally, let $m=\left(m_{1}, \cdots, m_{n}\right)$ be the vector of moments. $F_{3}$ maps $s$ onto $d=2 s / n-$ $m=F x$. Clearly, $F$ is a homeomorphism from $X$ onto $D=F(X)$. Since $X$ is compact, so is $D$. By Brouwer's Invariance of Domain Theorem [3], int $(D)=F(\operatorname{int}(X))$, and the boundary of $D, \dot{D}=F(\dot{X})$. Now, the original problem is equivalent to minimizing $\|\cdot\|_{2}$ on $D$. Since $F x=0$ has no roots in $X, 0 \notin D$. By the continuity of $\|\cdot\|_{2}$, compactness of $D$, and unimodality of $\|\cdot\|_{2}$ on $R^{n}$, $\|\cdot\|_{2}$ has a minimum only in $\dot{D}$.

Let

$$
\begin{aligned}
& X_{1}=\left\{x \in X: x_{1}=-1 \text { or } x_{n}=1 ; x_{i} \neq x_{i}, i \neq j\right\}, \\
& X_{2}=\left\{x \in X: x_{i}=x_{i} \text { for some } i \neq j\right\}, \\
& D_{1}=F\left(X_{1}\right), \quad D_{2}=F\left(X_{2}\right) .
\end{aligned}
$$

The boundary of $X$ is $\dot{X}=X_{1} \cup X_{2}$, and $\dot{D}=D_{1} \cup D_{2}$. It remains to show that if $\zeta \in D$ minimizes $\|\cdot\|_{2}$, then $\zeta \in D_{2}$, for, in that case, $F^{-1} \zeta \in X_{2}$ and corresponds to a degenerate formula. We will show that every deleted neighbourhood of $d \in D_{1}$ contains points $d+\Delta d$ such that $\|d+\Delta d\|_{2}<\|d\|_{2}$ and $d+\Delta d \in \operatorname{int}(D)$.

Consider first the case when $n$ is even. Let $\Delta d=\left(0, \cdots, 0, \Delta d_{n}\right) \in R^{n}$.

LEMMA 1. For every even integer $n>0$ there exists $\gamma<0$, depending on $n$ and $d$, such that $d+\Delta d \in \operatorname{int}(D)$ and $\|d+\Delta d\|_{2}<\|d\|_{2}$ whenever $d \in D_{1}$ and $\Delta d_{n} \in(\gamma, 0)$.

Proof. Let $d \in D_{1}$ be fixed but arbitrary. We have $s+\Delta s=F_{3}^{-1}(d+\Delta d)=$ $\left(F_{3}^{-1} d\right)+\Delta s$, where $\Delta s=\left(0, \cdots, 0, n \Delta d_{n} / 2\right) \in R^{n}$. From Newton's identities, $F_{2}^{-1}(s+\Delta s)=a+\Delta a$, where $\Delta a=\left(0, \cdots, 0,-\frac{1}{2} \Delta d_{n}\right)$. We can ensure that $F^{-1}(d+\Delta d)=F_{1}^{-1}(a+\Delta a) \in \operatorname{int}(X)$ by restricting $\Delta d$. To see this, let $p(d ; \cdot)$ be the monic $n$th degree polynomial whose zeros are given by $F^{-1} d$, and whose coefficients are, of course, given by $F_{2}^{-1} F_{3}^{-1} d$. We see that the increment $\Delta d_{n}$ in the $d_{n}$ coordinate of $d$ results in the addition of the constant $-\frac{1}{2} \Delta d_{n}$ to $p(d ; \cdot)$. Since the zeros of $p(d ; \cdot)$ are distinct and are all in $[-1,1]$, those of $p(d+\Delta d ; \cdot)$ will be in $(-1,1)$ provided that $\Delta d_{n}<0$ and $\left|\Delta d_{n}\right|$ is sufficiently small. There are exactly $n / 2$ local minima of $p(d ; \cdot)$ in $(-1,1)$ and $p(d ; \cdot)$ has negative values, say $u_{1}, \cdots, u_{n / 2}$, there. Let $\gamma_{1}=$ $2 \max \left\{u_{1}, \cdots, u_{n / 2}\right\}$. If $\Delta d_{n} \in\left(\gamma_{1}, 0\right)$, then $p(d+\Delta d ; \cdot)$ has $n$ real and distinct zeros in $(-1,1)$, and so $F^{-1}(d+\Delta d) \in \operatorname{int}(X)$, which implies $d+\Delta d \in \operatorname{int}(D)$.

Because $F^{-1} d \in X_{1}$,

$$
d_{n}=\frac{2}{n} \sum_{i=1}^{n} x_{i}^{n}-m_{n}>\frac{2}{n}-\frac{2}{n+1}>0 .
$$

It follows that $\|d\|_{2}^{2}-\|d+\Delta d\|_{2}^{2}>0$, if, and only if, $-\Delta d_{n}\left(\Delta d_{n}+2 d_{n}\right)>0$. Since $\Delta d_{n}<0$, we therefore require $\Delta d_{n}>-2 d_{n}$. Let $\gamma=\max \left\{\gamma_{1},-2 d_{n}\right\}<0$. Then $\Delta d_{n} \in(\gamma, 0)$ implies $d+\Delta d \in \operatorname{int}(D)$ and $\|d+\Delta d\|_{2}<\|d\|_{2}$.

It is somewhat more difficult to prove a similar lemma when $n$ is odd. For $x \in R^{n}$, define $S_{i}: R^{n} \rightarrow R$ by $S_{i} x=\sum_{i=1}^{n} x_{i}^{i}, j=1,2, \cdots, n$, and let $T_{\beta}=S_{n-1}+\beta S_{n}$ for all real $\beta$. For every constant $\mu$, let

$$
\begin{aligned}
& P_{\mu}^{-}=\left\{x \in R^{n}: x_{1}=-1, S_{1} x=\mu\right\}, \\
& P_{\mu}^{+}=\left\{x \in R^{n}: x_{n}=1, S_{1} x=\mu\right\}, \\
& P_{\mu}^{0}=\left\{x \in R^{n}: x_{1}=-1, x_{n}=1, S_{1} x=\mu\right\} .
\end{aligned}
$$

We first prove 
LEMMA 2. Let $n$ be odd.

(i) If $\beta=0$, then $T_{\beta} x \geqq 1$ for all $x \in P_{\mu}^{-} \cup P_{\mu}^{0} \cup P_{\mu}^{+}$, the equality holding only for a point of $X_{2}$ when $x \in P_{-1}^{-}$or $x \in P_{1}^{+}$; the inequality is strict if $x \in P_{\mu}^{0}$.

(ii) If $x \in P_{\mu}^{-} \cup P_{\mu}^{0}, \mu \leqq-1, \beta<0$, then $T_{\beta} x>1$.

(iii) If $x \in P_{\mu}^{+} \cup P_{\mu}^{0}, \mu \geqq 1, \beta>0$, then $T_{\beta} x>1$.

Proof. (i) If $\beta=0$, then $T_{\beta} x=S_{n-1} x$. When $x \in P_{\mu}^{-}, S_{n-1} x=1+\sum_{i=2}^{n} x_{i}^{n-1}=1$ if, and only if, $x_{2}=x_{3}=\cdots=x_{n}=0$. But then $\mu=-1$, and $(-1,0, \cdots, 0) \in X_{2}$. Otherwise $S_{n-1} x>1$. The other cases when $\beta=0$ are as trivial.

(ii) If $x \in P_{\mu}^{-}$, then

$$
\begin{aligned}
T_{\beta} x= & \left(\mu+1-x_{3}-\cdots-x_{n}\right)^{n-1}+\sum_{i=3}^{n} x_{i}^{n-1} \\
& +\beta\left(\mu+1-x_{3}-\cdots-x_{n}\right)^{n}+\beta \sum_{i=3}^{n} x_{i}^{n}+1-\beta \\
= & {[2+n \beta(\mu+1)] \sum_{i=3}^{n} x_{i}^{n-1}+\text { lower degree terms. } }
\end{aligned}
$$

It follows that the restriction of $T_{\beta}$ to $P_{\mu}^{-}$has a global minimum at a critical point provided that $2+n \beta(\mu+1)>0$. This is satisfied in view of the hypotheses for (ii). In order to find the values of $T_{\beta}$ at critical points, we use a Lagrange multiplier. Then

$$
\frac{\partial}{\partial x_{i}}\left[T_{\beta} x-\lambda\left(S_{1} x-\mu\right)\right]=0, \quad j=2,3, \cdots, n
$$

implies

$$
(n-1) x_{i}^{n-2}+n \beta x_{i}^{n-1}=\lambda, \quad j=2,3, \cdots, n .
$$

The additional equation to be satisfied is

$$
x_{2}+x_{3}+\cdots+x_{n}=\mu+1 \text {. }
$$

Suppose that $x_{2}^{*}, \cdots, x_{n}^{*}, \lambda^{*}$ is a solution of (2.3a) and (2.3b), and denote the critical point $\left(-1, x_{2}^{*}, \cdots, x_{n}^{*}\right)$ by $x^{*}$. Then, since $\beta<0$ and $\mu+1 \leqq 0$ by hypothesis, we must have $\lambda^{*} \leqq 0$. Otherwise, Eqs. (2.3a) could only be satisfied by positive numbers $x_{2}^{*}, \cdots, x_{n}^{*}$, and this would contradict (2.3b). Multiplying the $j$ th equation in (2.3a) by $x_{i}^{*}$ and summing, $j=2, \cdots, n$, we find, with (2.3b), that

$$
(n-1)\left(S_{n-1} x^{*}-1\right)+n \beta\left(S_{n} x^{*}+1\right)=\lambda^{*}(\mu+1),
$$

and upon rearrangement,

$$
T_{\beta} x^{*}=S_{n-1} x^{*}+\beta S_{n} x^{*}=1+(1 / n)\left[\lambda^{*}(\mu+1)-\beta n+S_{n-1} x^{*}-1\right]>1
$$

at every critical point since $\beta<0, \lambda^{*} \leqq 0, \mu+1 \leqq 0$ and $S_{n-1} x^{*}>1$. This is the required result.

Proofs of the cases when $x \in P_{\mu}^{0}$ and $x \in P_{\mu}^{+}$are very similar to the above.

We are now ready to proceed with the proof of

LeMma 3. Let $\Delta d=\left(0, \cdots, 0, \Delta d_{n-1}, \Delta d_{n}\right)$ and $\Delta d_{n}=\beta \Delta d_{n-1}$. For every odd integer $n>0$ and every $d \in D_{1}$, there exist $\beta$ and $\gamma(\beta)<0$, depending on $n$ and $d$, 
such that $d+\Delta d \in \operatorname{int}(D)$ and $\|d+\Delta d\|_{2}<\|d\|_{2}$ whenever $d \in D_{1}$ and $\Delta d_{n-1} \in$ $(\gamma, 0)$.

Proof. For any $d \in D$, let $p(d ; \cdot)$ be the monic $n$th degree polynomial with zeros given by $F^{-1} d$ and coefficients given by $F_{2}^{-1} \cdot F_{3}^{-1} d$ as in Lemma 1 . Now, $s+\Delta s=$ $F_{3}^{-1}(d+\Delta d)=F_{3}^{-1} d+n \Delta d / 2$, so that $\Delta s=n \Delta d / 2$. Using Newton's identities, we obtain $a+\Delta a=F_{2}^{-1}(s+\Delta s)$ with

$$
\Delta a=\left(0, \cdots, 0,-\frac{\Delta s_{n-1}}{n-1}, \frac{s_{1} \Delta s_{n-1}}{n-1}-\frac{\Delta s_{n}}{n}\right) \text {. }
$$

It follows that

$$
\begin{aligned}
\Delta p(d ; t) & =-\frac{\Delta s_{n-1}}{n-1} t+\frac{s_{1}}{n-1} \frac{\Delta s_{n-1}}{n-1}-\frac{\Delta s_{n}}{n} \\
& =-\frac{\Delta s_{n-1}}{n-1}\left(t-s_{1}+\frac{n-1}{n} \beta\right) .
\end{aligned}
$$

The effect, then, of the change $\Delta d$ in $d$, is to add the linear polynomial $\Delta p(d ; \cdot)$ to $p(d ; \cdot)$, thereby causing a shift in the zeros of $p(d ; \cdot)$. The magnitude of this shift depends on more variables than in the even case.

Let

$$
\begin{aligned}
& X_{1}^{-}=\left\{x \in X_{1}: x_{1}=-1, x_{n}<1\right\}, \\
& X_{1}^{+}=\left\{x \in X_{1}: x_{1}>-1, x_{n}=1\right\}, \\
& X_{1}^{0}=\left\{x \in X_{1}: x_{1}=-1, x_{n}=1\right\} .
\end{aligned}
$$

Clearly $X_{1}=X_{1}^{-} \cup X_{1}^{0} \cup X_{1}^{+}$. Suppose first that $d \in D_{1}$ and that $F^{-1} d \in X_{1}^{-}$. Then, $p(d ;-1)=0, p(d ; 1)>0$, and all zeros of $p(d ; \cdot)$ are real, distinct and in $[-1,1)$. Now, $d+\Delta d \in \operatorname{int}(D)$ if, and only if, $p(d ; \cdot)+\Delta p(d ; \cdot)$ has $n$ real, distinct zeros in $(-1,1)$. The following conditions are sufficient for the real zeros of $p(d ; \cdot)+$ $\Delta p(d ; \cdot)$ to lie in $(-1,1)$ :

$$
\begin{aligned}
\text { (i) } & \Delta d_{n-1}<0, \\
\text { (ii) } & \Delta p(d ;-1)<0, \\
\text { (iii) } & \Delta p(d ; 1)>-p(d ; 1) .
\end{aligned}
$$

To verify, we note that if (i) and (ii) hold, then $\Delta p(d ; \cdot)$ has positive slope and $\Delta p(d ; \cdot)$ $<0$ on $(-\infty,-1]$. Also, $p(d ; \cdot)$ is of odd degree and its smallest zero is -1 . Hence, $p(d ; \cdot)+\Delta p(d ; \cdot)<0$ on $(-\infty,-1]$ and has no zeros there. If (i) and (iii) hold, then the positive slope of $\Delta p(d ; \cdot)$ implies that $\Delta p(d ; \cdot)>-p(d ; 1)$ on $[1, \infty)$. Since $p(d ; \cdot)$ is monic and its largest zero is less than unity, $p(d ; \cdot)>p(d ; 1)>0$ on $[1, \infty)$. It follows that $p(d ; \cdot)+\Delta p(d ; \cdot)>0$ on $[1, \infty)$ and has no zeros there either.

Using (2.4), we may rewrite (2.5) as

(i) $\Delta s_{n-1}<0$,

(ii) $\beta<n\left(s_{1}+1\right) /(n-1)$,

(iii) $\Delta s_{n-1}\left(1-s_{1}+(n-1) \beta / n\right)<(n-1) p(d ; 1)$. 
In view of the simple relationship between $\Delta s$ and $\Delta d$, namely $\Delta d=2 \Delta s / n,(2.6)$ could be written as a condition on $\Delta d_{n-1}$ and $\beta$, but the introduction of the factor $n / 2$ that would result, only complicates the expressions that arise. We therefore postpone it until later.

Since the coefficients of $t$ in $\Delta p(d ; t)$ are continuous functions of $\Delta s_{n-1}$, and the zeros of $p(d ; \cdot)+\Delta p(d ; \cdot)$ are continuous functions of the coefficients, it follows that, for a given $\beta$ and $d \in D_{1}, p(d ; \cdot)+\Delta p(d ; \cdot)$ has only real and distinct zeros provided that $\left|\Delta s_{n-1}\right|$ is sufficiently small. Then, assuming (2.6)(i) holds, there exists $\delta_{1}(\beta)<0$ such that

$$
\text { (iv) } \delta_{1}(\beta)<\Delta s_{n-1}<0
$$

implies that the zeros of $p(d ; \cdot)+\Delta p(d ; \cdot)$ are real and distinct.

All of the conditions in (2.6) guarantee that $d+\Delta d \in \operatorname{int}(D)$.

Our further objective is to ensure that $\|d+\Delta d\|_{2}<\|d\|_{2}$. Using the definitions in the statement of this lemma, a straightforward calculation shows that

$$
\|d+\Delta d\|_{2}^{2}-\|d\|_{2}^{2}=\frac{4}{n^{2}} \Delta s_{n-1}\left[\Delta s_{n-1}\left(1+\beta^{2}\right)+2\left(s_{n-1}+\beta s_{n}-1\right)\right],
$$

where we have used the fact that $d_{n-1}=2 s_{n-1} / n-2 / n$. This norm-difference is negative if

$$
\Delta s_{n-1}>-2\left(s_{n-1}+\beta s_{n}-1\right) /\left(1+\beta^{2}\right)=-2\left(T_{\beta} F^{-1} d-1\right) /\left(1+\beta^{2}\right),
$$

since $\Delta s_{n-1}<0$ by (2.6)(i). But then, also the right-hand side of (2.7) must be negative, so that we need

$$
T_{\beta} F^{-1} d-1>0
$$

so as not to contradict (2.6)(i). This is a condition on the choice of $\beta$ and can be satisfied by using Lemma 2 . Then, (2.7) supplies a lower bound for $\Delta s_{n-1}$; we denote it by $\delta_{2}(\beta)$.

We must now reconcile the conditions expressed in (2.6), (2.7) and (2.8), and, to this end, we distinguish three cases, assuming right away that (2.6)(i) holds, that is, $\Delta s_{n-1}<0$ :

(i) $s_{1}=S_{1} F^{-1} d \leqq-1$. We choose $\beta$ according to (2.6)(ii). Since $s_{1}+1 \leqq 0$, the $\beta$ is negative. Now $x=F^{-1} d \in P_{s_{1}}^{-}$with $s_{1} \leqq-1, \beta<0$, so that by Lemma 2(ii), $T_{\beta} x=T_{\beta} F^{-1} d>1$. Hence, (2.8) holds. In addition, we take

$$
\beta>n\left(S_{1} F^{-1} d-1\right) /(n-1) \text {. }
$$

This means that the coefficient of $\Delta s_{n-1}$ in (2.6)(iii) is positive. Since $p(d ; 1)>0$, (2.6)(iii) does not restrict $\Delta s_{n-1}<0$. With $\beta$ satisfying both (2.6)(ii) and (2.9), we choose

$$
0>\Delta s_{n-1}>\max \left\{\delta_{1}(\beta), \delta_{2}(\beta)\right\}=\delta(\beta),
$$

and set $\gamma(\beta)=2 \delta(\beta) / n$. The lemma is proved for this case.

It will be of importance in the next section to observe that conditions (2.6)(ii) and (2.9) can be satisfied without choosing $\beta$ outside the interval $[-n, 0)$. This is because $F^{-1} d \in X_{1}^{-}$, and therefore $S_{1} F^{-1} d>-n$.

(ii) $s_{1}=S_{1} F^{-1} d \in(-1,1]$. Now $s_{1}+1>0$, and we satisfy both (2.6)(ii) and, 
by Lemma 2(i), (2.8), by taking $\beta=0$. The coefficient of $\Delta s_{n-1}$ in (2.6)(iii) is nonnegative, hence (2.6)(iii) does not restrict $\Delta s_{n-1}$. Consequently, we must choose

$$
0>\Delta s_{n-1}>\max \left\{\delta_{1}(0), \delta_{2}(0)\right\}=\delta(0) \text {, or } 0>\Delta d_{n-1}>\gamma(0) \text {. }
$$

(iii) $s_{1}=S_{1} F^{-1} d>1$. As in the previous case, the choice $\beta=0$ satisfies both (2.6)(ii) and (2.8). Let

$$
\delta_{3}=(n-1) p(d ; 1) /\left(1-s_{1}\right)<0 .
$$

Now, (2.6)(iii), (iv) and (2.7) are satisfied when

$$
0>\Delta s_{n-1}>\max \left\{\delta(0), \delta_{3}\right\}=\delta,
$$

or

$$
0>\Delta d_{n-1}>\gamma(0), \quad \gamma(0)=2 \delta / n .
$$

We have exhibited a choice of $\beta$ and a $\gamma(\beta)$ such that $d+\Delta d \in \operatorname{int}(D)$ and $\|d+\Delta d\|_{2}<\|d\|_{2}$ whenever $\Delta d_{n-1} \in(\gamma(\beta), 0)$, and $d \in D_{1}$ is such that $F^{-1} d \in$ $X_{1}^{-}$. Furthermore, it is not necessary to use a $\beta$ outside $[-n, 0]$. The cases when $F^{-1} d \in X_{1}^{0}$ or $X_{1}^{+}$are dealt with in a similar way, some small changes in (2.6)(i), (ii) and (iii) being necessary.

When all cases are combined, one finds that $\beta$ need not be chosen outside $[-n, n]$.

We conclude that $\|d\|_{2}$ cannot attain its minimum value on $D_{1}$, rather this occurs either in $\operatorname{int}(D)$ or on $D_{2}$. We therefore have the

THEOREM 1. For $n=8$ and $n>9$ every almost-interpolatory Chebyshev quadrature formula is degenerate.

3. Generalizations. It is possible to generalize the preceding results in several directions. One of these is the consideration of other than constant weight functions in (1.1).

Let $W$ be the set of all weight functions on $[-1,1]$ having unit integral. This normalization is convenient. Let $\left\{\mu_{0}(\omega), \mu_{1}(\omega), \cdots\right\}$ be the moment sequence of $\omega \in W$, that is,

$$
\mu_{i}(\omega)=\int_{-1}^{1} \omega(x) x^{i} d x, \quad j=0,1, \cdots, n .
$$

The normalization implies that $\mu_{0}(\omega)=1$ for all $\omega \in W$. For every fixed positive integer $n$, we shall use $\mu(\omega)$ to denote the moment vector $\left(\mu_{1}(\omega), \cdots, \mu_{n}(\omega)\right)$. Now, it is required to approximate $\int_{-1}^{1} \omega(x) f(x) d x$ by $c \sum_{i=1}^{n} f\left(x_{i}\right)$, where $x=\left(x_{1}, \cdots, x_{n}\right)$ $\in X$, and the approximation has no error when $f \in \mathcal{P}_{n}$. Taking $f(x) \equiv 1$, we find $\mu_{0}(\omega)=c n$, and therefore, choose $c=1 / n$. Taking $f(x)=x^{i}, j=1,2, \cdots, n$, we again obtain residuals

$$
d_{i}=\frac{1}{n} \sum_{i=1}^{n} x_{i}^{i}-\mu_{i}(\omega), \quad j=1,2, \cdots, n,
$$

as the components of the residual vector $d \in R^{n}$. The resulting quadrature formula is interpolatory if there exists $x \in X$ such that $d=0$. We are thus led to the search for minimizers of $\|d\|_{2}$.

Let $F_{\omega}: X \rightarrow R^{n}$ be defined by 


$$
F_{\omega} x=\left\{\begin{array}{c}
\frac{1}{n} \sum_{i=1}^{n} x_{i}-\mu_{1}(\omega) \\
\vdots \\
\frac{1}{n} \sum_{i=1}^{n} x_{i}^{n}-\mu_{n}(\omega) .
\end{array}\right.
$$

As before, $F_{\omega}$ is a homeomorphism from $X$ onto $D(\omega)=F_{\omega}(X)$. We note that if $\omega_{1}$ and $\omega_{2} \in W$ then

$$
F_{\omega_{2}} x=F_{\omega_{1}} x+\mu\left(\omega_{1}\right)-\mu\left(\omega_{2}\right) .
$$

It follows that a change in weight function results merely in a translation of $D\left(\omega_{1}\right)$ to $D\left(\omega_{2}\right)$. Suppose that $0 \notin D\left(\omega_{1}\right)$ and $0 \notin D\left(\omega_{2}\right)$, and let $F_{\omega_{1}}\left(X_{2}\right)=D_{2}\left(\omega_{1}\right)$ be the "side" of $D\left(\omega_{1}\right)$ closest to 0 , so that $\|d\|_{2}$ is minimized on $D_{2}\left(\omega_{1}\right)$, corresponding to a degeneracy. The translation of $D\left(\omega_{1}\right)$ onto $D\left(\omega_{2}\right)$ can easily be such that now $D_{1}\left(\omega_{2}\right)$ is the side of $D\left(\omega_{2}\right)$ closest to 0 . This means that the proofs of Lemmas 1 and 3 will extend to other weight functions only if the choice of weight function is quite restricted. Those proofs are in two parts. The first part shows that $d+\Delta d \in \operatorname{int}(D)$ if $\|\Delta d\|_{2}$ is sufficiently small, and is independent of the weight function. The second part shows that $\|d+\Delta d\|_{2}<\|d\|_{2}$ and does depend on the weight function. For $n$ even, $\Delta d_{n}<0$ is necessary for the first part of the proof. Then, in the second part, we obtain $\|d+\Delta d\|_{2}<\|d\|_{2}$ only if $\Delta d_{n}>-2 d_{n}$. This is possible only if $d_{n}>0$. But, on $D_{1}(\omega), d_{n}>1 / n-\mu_{n}(\omega)$, and this is the best possible bound. Hence, $\|d+\Delta d\|_{2}$ $<\|d\|_{2}$ only if $\mu_{n}(\omega) \leqq 1 / n$. In Lemma 1 , with the scale change due to normalization, we have $\omega \equiv \frac{1}{2}$, and $\mu_{n}\left(\frac{1}{2}\right)=1 /(n+1)<1 / n$.

In the odd case, using the more general $F_{\omega}$, we find that

$\|d+\Delta d\|_{2}^{2}-\|d\|_{2}^{2}=\frac{1}{n^{2}} \Delta s_{n-1}\left[\Delta s_{n-1}\left(1+\beta^{2}\right)+2\left(s_{n-1}+\beta s_{n}-n \beta \mu_{n}(\omega)-n \mu_{n-1}(\omega)\right)\right]$. if

As in the proof of Lemma 3, $\Delta s_{n-1}<0$ implies that this norm-difference is negative

$$
\Delta s_{n-1}>\frac{-2\left[s_{n-1}+\beta s_{n}-n \beta \mu_{n}(\omega)-n \mu_{n-1}(\omega)\right]}{1+\beta^{2}}
$$

and the right-hand side must then be negative as well, so that we need, as a condition on $\beta$,

$$
s_{n-1}+\beta s_{n}-n \beta \mu_{n}(\omega)-n \mu_{n-1}(\omega)>0 .
$$

Inequalities (3.1) and (3.2) are the generalizations of (2.7) and (2.8). We may rewrite (3.2) as

$$
\beta \mu_{n}(\omega)+\mu_{n-1}(\omega)<T_{\beta} F_{\omega}^{-1} d / n .
$$

As in Lemma 3, $\beta$ can always be selected so that $T_{\beta} F_{\omega}^{-1} d>1$, and (3.2) will be satisfied if

$$
\beta \mu_{n}(\omega)+\mu_{n-1}(\omega) \leqq 1 / n .
$$

Since we may restrict $\beta$ to $[-n, n]$, we find when $\beta=0$ that 


$$
\mu_{n-1}(\omega) \leqq 1 / n \text { and }\left|\mu_{n}(\omega)\right| \leqq\left[1 / n-\mu_{n-1}(\omega)\right] /|\beta|
$$

otherwise. As a $\beta$-free condition on $\mu_{n-1}(\omega)$ and $\mu_{n}(\omega)$, we may therefore take

$$
\mu_{n-1}(\omega) \leqq 1 / n, \quad\left|\mu_{n}(\omega)\right| \leqq 1 / n^{2}-\mu_{n-1}(\omega) / n .
$$

If we replace (2.7) by (3.1) and (3.3) holds, then the proof of Lemma 3 can be applied as it stands to complete the proof of

THEOREM 2. Let $n$ and $\omega \in W$ be such that the interpolatory Chebyshev quadrature does not exist. If $n$ is even and $\mu_{n}(\omega) \leqq 1 / n$, or if $n$ is odd and (3.3) holds, then the corresponding almost-interpolatory formula is degenerate.

Another generalization is to minimize $\|d\|_{p}, 1 \leqq p<\infty$. When $n$ is even it is trivial to extend the argument from Lemma 1 , with $\mu_{n}(\omega) \leqq 1 / n$, to the $p$-norm.

When $n$ is odd it seems necessary to impose a more restrictive condition than (3.3) on the weight function. It is sufficient to require that

$$
\mu_{n-1}(\omega) \leqq 1 / n, \quad \mu_{n}(\omega)=0 .
$$

With this assumption, we find that $d_{n-1}>0$ for all $d \in D_{1}(\omega)=F_{\omega}\left(X_{1}\right)$. If $d \in$ $D_{1}(\omega), F_{\omega}^{-1} d \in X_{1}^{-}$and $S_{1} F_{\omega}^{-1} d \leqq-1$, then, by Lemma 2 ,

$$
S_{n-1} F_{\omega}^{-1} d+\beta S_{n} F_{\omega}^{-1} d>1, \quad \forall \beta<0 .
$$

In terms of the components of $d$, this is equivalent to

$$
d_{n-1}+\beta d_{n}>1 / n-\mu_{n-1}(\omega), \quad \forall \beta<0 .
$$

By (3.4), it follows that $d_{n-1}+\beta d_{n}>0, \forall \beta<0$. Since $d_{n-1}>0$, this implies $d_{n}$ $<0$. Now,

$$
\|d+\Delta d\|_{p}^{p}-\|d\|_{p}^{p}=\left|d_{n-1}+\Delta d_{n-1}\right|^{p}-\left|d_{n-1}\right|^{p}+\left|d_{n}+\beta \Delta d_{n-1}\right|^{p}-\left|d_{n}\right|^{p} .
$$

This is negative if

$$
0>\Delta d_{n-1}>\max \left\{-2 d_{n-1}, 2 d_{n} / \beta\right\},
$$

and (3.5) replaces (2.7) in Lemma 3 , which now applies verbatim for $S_{1} F_{\omega}^{-1} d \leqq-1$.

If $S_{1} F_{\omega}^{-1} d>-1$, we again choose $\beta=0$ and then $\|d+\Delta d\|_{p}-\|d\|_{p}<0$ if $0>\Delta d_{n-1}>-2 d_{n-1}$, and this replaces (2.7) in Lemma 3 .

If $F_{\omega}^{-1} d \in X_{1}^{+}$, and $S_{1} F_{\omega}^{-1} d \geqq 1$, then Lemma 2 can again be employed to show that now $d_{n}>0$ while $\beta>0$. In this case, a norm-decrease results if

$$
0>d_{n-1}>\max \left\{-2 d_{n-1},-2 d_{n} / \beta\right\} \text {. }
$$

If $S_{1} F_{\omega}^{-1} d<1$, the choices $\beta=0$ and $0>d_{n-1}>-2 d_{n-1}$ again ensure a normdecrease.

When $F_{\omega}^{-1} d \in X_{1}^{0}$, the lower bounds on $\Delta d_{n-1}$ are obtained in a similar fashion.

The proof does not extend to the case $p=\infty$.

We combine the even and odd cases in

THEOREM 3. Let $\omega \in W$ be a normalized weight function on $[-1,1]$ whose moment sequence $\left\{\mu_{i}(\omega)\right\}_{i=0}^{\infty}$ has the property

$$
\mu_{0}(\omega)=1, \quad \mu_{2 j-1}(\omega)=0, \quad \mu_{2 j}(\omega) \leqq 1 / 2 j, \quad j=1,2, \cdots .
$$

If for some positive integer $n$ the corresponding interpolatory Chebyshev quadrature 
formula does not exist, then the almost-interpolatory formula defined by a minimizer of $\left\|F_{\omega} x-\mu(\omega)\right\|_{p}, 1 \leqq p<\infty, \mu(\omega)=\left(\mu_{1}(\omega), \cdots, \mu_{n}(\omega)\right)$, is degenerate.

Acknowledgment. The author gratefully acknowledges the assistance of P. J. Taylor through many discussions, and the referee's encouragement to generalize the results of Section 2. Thanks are also due to the University of Calgary for the granting of sabbatical leave during which this work was completed, and the Mathematics Department of the University of Southampton as well as the Institut für Numerische und Instrumentelle Mathematik of the University of Münster for the provision of the necessary facilities.

Department of Mathematics, Statistics, and Computing Science The University of Calgary Calgary, Alberta, Canada

1. R. E. Barnhill, J. E. Dennis, JR. \& G. M. Nielson, "A new type of Chebyshev quadrature," Math. Comp., v. 23, 1969, pp. 437-441. MR 39 \#3698.

2. S. N. BERNSTEIN, "On quadrature formulas of Cotes and Chebyshev," Dokl. Akad. Nauk SSSR, v.14, 1937, pp. 323-326. (Russian)

3. W. Hurewicz \& H. Wallmann, Dimension Theory, Princeton Math. Series, vol. 4, Princeton Univ. Press, Princeton, N.J., 1941. MR 3, 312.

4. V. I. KRYLov, Approximate Calculation of Integrals, Fizmatgiz, Moscow, 1959; English transl., Macmillan, New York, 1962. MR 22 \#2002; MR 26 \#2008. 